\title{
Clinical features of paediatric acute-onset neuropsychiatric syndrome: findings from a case- control study
}

Eva Hesselmark and Susanne Bejerot

\section{Background}

Paediatric acute-onset neuropsychiatric syndrome (PANS), an umbrella term that includes PANDAS (paediatric autoimmune neuropsychiatric disorders associated with streptococcal infections) is suggested to be a psychiatric disorder of autoimmune aetiology. PANS is characterised by an acute onset of obsessivecompulsive disorder or restricted eating with multiple comorbid symptoms. The specificity of the PANS criteria is not fully understood.

\section{Aims}

To describe a cohort of patients with PANS and to determine if PANS features relating to symptoms, onset and course are more common in PANS than in other psychiatric conditions.

\section{Method}

A case-control study comparing patients with interview-confirmed PANS with patients with suspected PANS and patients with a psychiatric condition but with no suspicion of PANS. Validated and non-validated measures of symptoms, onset and episodic course were used.

\section{Results}

Illness in patients with interview-confirmed PANS featured an episodic course and multiple symptoms present at onset compared with the psychiatric controls. However, individuals with interview-confirmed PANS did not present a specific symptom profile.

\section{Conclusions}

PANS may be a distinct clinical entity featuring an acute onset, an episodic course and multiple symptoms at onset.

\section{Declaration of interest}

None.

\section{Keywords}

Pediatric acute-onset neuropsychiatric syndrome; pediatric autoimmune neuropsychiatric disorders associated with streptococcal infections; diagnostic criteria; obsessive-compulsive disorder.

\section{Copyright and usage}

(c) The Royal College of Psychiatrists 2019. This is an Open Access article, distributed under the terms of the Creative Commons Attribution-NonCommercial-NoDerivatives licence (http://creativecommons.org/licenses/by-nc-nd/4.0/), which permits noncommercial re-use, distribution, and reproduction in any medium, provided the original work is unaltered and is properly cited. The written permission of Cambridge University Press must be obtained for commercial re-use or in order to create a derivative work.
Paediatric acute-onset neuropsychiatric syndrome (PANS) and paediatric autoimmune neuropsychiatric disorders associated with streptococcal infections (PANDAS) are specific obsessive-compulsive disorder (OCD) phenotypes that include acute onset of OCD symptoms, restricted eating or tics comorbid with multiple psychiatric and neurological symptoms ${ }^{1,2}$ (see supplementary Table 1 available at https://10.1192/bjo.2019.10 for full criteria of both disorders). Although the criteria themselves do not stipulate autoimmunity, both disorders are thought to be autoimmune encephalopathies. The exact pathophysiology in PANS and PANDAS is currently unknown; however, treatment with immunomodulatory drugs is recommended ${ }^{3}$ although, their efficacy remains unclear. ${ }^{4}$

\section{Criteria for PANDAS and PANS}

The criteria for PANDAS were first described by Swedo and her colleagues as research criteria in $1998 .{ }^{1}$ PANDAS includes a temporal association between streptococcal infection and psychiatric symptom onset or exacerbation. PANS criteria, established in $2012,{ }^{2}$ are not defined by an aetiology and thus do not require a preceding infection. Both PANS and PANDAS are characterised by the acute onset of symptoms.

In addition to acute onset, PANDAS is described as having an 'episodic course', whereas this is not stated in the PANS criteria. However, in a clinical description of 47 patients at a clinic dedicated to PANS, $89 \%$ were reported to have a 'relapsing/remitting' (i.e. episodic) course. ${ }^{5}$ Moreover, two recent treatment studies of PANS used 'number of flares' as the main outcome measure. ${ }^{6,7}$
Therefore, the existence of an episodic course, an important clinical feature of PANS as well as PANDAS, needs to be further investigated.

Beyond the diagnostic criteria of OCD and/or tics, Swedo and colleagues also reported a high frequency of several other symptoms in the first 50 individuals diagnosed with PANDAS, including emotional lability, deterioration of school performance, personality change, bedtime fears and rituals, fidgetiness, separation anxiety, irritability, tactile/sensory defensiveness, impulsivity/distractibility, deterioration in handwriting, choreiform movements, oppositional/defiant behaviour and nightmares. ${ }^{1}$ All these symptoms were subsequently incorporated as supplementary criteria for PANS. Furthermore, sleep disorder and urinary issues were also included as signs of PANS. ${ }^{2}$ Some of these symptoms are common in many psychiatric disorders, including Tourette syndrome and OCD, and therefore their specificity as diagnostic criteria of PANS remain unknown. Notably, PANS criteria do not only stipulate the presence of these symptoms but also a dramatic onset or exacerbation of multiple and concomitant symptoms.

PANS and PANDAS are increasingly recognised in the clinic and multiple parent interest groups are presently active in several European countries and the USA 2015 consensus paper for the diagnostic work-up recommended a broad assessment including psychiatric, immunological and infectious disease evaluation. ${ }^{8}$ However, some parts of these recommendations (i.e. the blood tests recommended) have been criticised for not being based on published patient data. ${ }^{9}$ Furthermore, the National Institute for Health and Care Excellence guidelines on OCD mention PANDAS, but do 
not provide a comprehensive description or any clinical guidelines. To summarise, PANS and PANDAS remain disorders that lack a global agreement on the symptomatology, onset and course. Despite this, PANS and PANDAS are used clinically to classify and treat patients.

\section{Aims}

The aims of this study are:

(a) to describe symptoms in a cohort of patients with PANS or PANDAS, and compare the symptom frequency with patients with suspected PANS or PANDAS and with that of patients with other psychiatric conditions;

(b) to determine if an episodic course is more common in patients with PANS or PANDAS than in patients with suspected PANS or PANDAS, or in patients with other psychiatric conditions;

(c) to determine if patients with PANS or PANDAS experience more symptoms at onset than patients with suspected PANS or PANDAS, or patients with other psychiatric conditions.

\section{Method}

\section{Study design}

This is a case-control study that compares three groups of patients: patients with interview-confirmed PANS, patients with suspected PANS and psychiatric patients who never were suspected of having PANS. PANS is defined as an umbrella term that includes PANDAS, ${ }^{2}$ therefore we use the term PANS when referring to patients with both PANS and PANDAS further on in this paper.

\section{Participants and recruitment}

In the current study, we used two methods of recruitment. First, we wanted to recruit a group of patients with a high probability of fulfilling criteria for PANS. We therefore invited all Swedish patients who had taken the Cunningham Panel, ${ }^{10-15}$ a blood test aimed to diagnose PANS and PANDAS (the Swedish name for this panel is 'The PANDAS-Panel'). We have previously published a paper that questions the diagnostic accuracy of the Cunningham Panel. ${ }^{16}$ The panel is costly and must be ordered by a physician, thus we concluded that patients who had been tested with this panel were suspected of having PANS. In this study the Cunningham Panel was solely used as a recruitment strategy and was not used to diagnose any participants with PANS. Eligibility to participate was based on having taken the test and not on the test results.

Participants in 'the Cunningham Panel sample' were enrolled through Wieslab, the Swedish laboratory that administers the Cunningham Panel. All patients who had taken the panel $(n=154)$ were invited, 53 of whom consented to participate in the study. They were all assessed by S.B., a senior psychiatrist, and E.H., trained in psychology, between June 2014 and January 2016. These participants have already been described in a study of the diagnostic properties of the Cunningham Panel for diagnosing PANS. ${ }^{16}$

Second, in order to obtain a credible comparison group of psychiatric patients without PANS, we advertised for volunteers at a psychiatric clinic in Örebro, Sweden. Inclusion criteria for 'the Örebro sample' $(n=36)$ was to have a psychiatric disorder requiring specialist care and to be age and gender matched to a participant in the Cunningham Panel sample. The Örebro sample was assessed by S.B. or Machi Cleanthous, a senior child and adolescent psychiatrist, and E.H. or Jasmina Popaja, a licensed clinical psychologist. Exclusion criteria for all participants were being older than 40 years of age and not speaking Swedish.

\section{Procedure}

All participants were interviewed on one occasion, either at various psychiatric facilities or in their homes. Participants unable to complete the interview independently were assisted by a parent or a caretaker. In the current paper, we refer all responses from the interview to be 'reported by participants'.

\section{Diagnostic work-up and classification}

The aim of the diagnostic work-up was to document the psychiatric and medical history of the participants and to classify them in regard to their PANS/PANDAS status, associated symptoms, episodic course, acute onset and symptom load at onset. The full procedure has also been described in a previous paper. ${ }^{16}$

PANS diagnosis was determined by careful assessment of current and previous signs and symptoms. The nature of the onset and the progression were described in retrospect during our interview. At the beginning of each interview, participants were asked about their current main psychiatric problems. They were also asked to describe the onset and course of the disorder. After this followed the use of standard psychiatric interviews and measures for psychiatric diagnostics, as well as structured interviews of PANS symptoms developed for use in this study. After each completed interview, the two participating clinicians conferred to determine if the participant fulfilled the required diagnostic criteria for PANS.

Acute onset was defined as a rapid progress of symptoms that peaked within $72 \mathrm{~h}$ after onset. Classification of PANS was into one of three groups: (a) interview-confirmed PANS, comprising all participants who fulfilled criteria of PANS according to the clinical assessment (IC-PANS group); (b) suspected (but not confirmed) PANS, comprising participants from the Cunningham Panel Sample who did not fulfil PANS criteria (S-PANS group); and (c) no PANS, comprising participants recruited as the psychiatric comparison sample who did not fulfil PANS criteria (never-PANS group). See supplementary Fig. 1 for a flow chart of the inclusion process.

The PANS criteria state that the symptoms should not be better explained by a known disorder such as Sydenham chorea, systemic lupus erythematosus, Tourette disorder or others. ${ }^{2}$ None of the participants had previous diagnoses of Sydenham chorea or systemic lupus erythematosus. Some participants fulfilled the criteria for Tourette disorder, but Tourette disorder did not better explain the non-tic symptoms of any of the participants in the study.

\section{Measures}

\section{Standard psychiatric interviews and measures}

The Mini International Neuropsychiatric Interview (MINI, version 6) ${ }^{17}$ is a structured interview for assessing multiple present and previous psychiatric diagnoses. In the current study, we used the MINI (Version 6) for adults and the MINI-KID ${ }^{18}$ (version 6) for children. The MINI-KID includes several items not included in the MINI for adults. To ensure the assessments between adults and children were compatible, we decided to also include the MINI-KID modules of the following diagnoses in the interview of the adult participants: separation anxiety, specific phobias, Tourette syndrome/tics, attention-deficit hyperactivity disorder, conduct disorder and oppositional defiant disorder.

The Yale-Brown Obsessive Compulsive Scale (Y-BOCS) and the Children's Yale-Brown Obsessive Compulsive Scale (CY-BOCS) ${ }^{19}$ are clinically administered instruments for assessing severity of OCD. It ranges from 0 to 40 points, with a higher score indicate higher severity. 
The Clinical Global Impression-Severity (CGI-S $)^{20}$ is a oneitem, clinician-rated measure of global severity. It ranges from one to seven points, with a high score indicating a high severity and a score of four corresponding to 'moderately ill'. In the current study, the CGI-S was conducted at the end of the clinical interview.

The clinical assessment included four sections of the Wechsler intelligence scales for adults (WAIS-IV) ${ }^{21}$ or children (WISCIII); ${ }^{22}$ block design, letter number sequencing, digit symbol coding and digit span. A full-scale IQ of each participant was estimated using the mean of the four scaled scores available and multiplying them by 11 .

\section{Biological measures}

In order to get a rough marker of current inflammation or infection, we also measured C-reactive protein in plasma and erythrocyte sedimentation rate in blood. Both these analyses were conducted according to standard clinical procedures at the time of assessment. Height and weight were reported during interview and used to estimate body mass index (BMI).

\section{Measure of PANS symptoms}

PANS Scale- $\mathrm{R}$ is an unpublished structured interview (Leckman, 2014, personal communication) based on the diagnostic criteria for PANS. ${ }^{2}$ To assess the sudden onset, the following open-ended question was presented to the patient 'Describe the onset of the PANS symptoms, was it sudden or gradual?' Criterion I (i.e. presence of OCD or an eating disorder) is assessed with six obsessivecompulsive-related items each assessing both obsessions and compulsions related to five common obsessive-compulsive symptom dimensions (contamination, causing harm, sexual or religious, symmetry, hoarding and other) and one item on eating restriction. Criterion II (i.e. the concurrent presence of additional neuropsychiatric symptoms for PANS) is determined through the assessment of 21 items corresponding to the seven symptom categories of criterion II (see supplementary Table 1 for a list of symptoms). Each item is rated as 'ever present' and/or 'currently present'. Furthermore, the onset date and whether a clinician verifies the symptom is recorded for each item. Criterion III (exclusion of other possible causes for the symptoms) is not assessed by this instrument. In this report we used the 'ever present' item for all 28 symptoms and a global severity score (0-100) for PANS-related symptoms.

\section{Measures of disorder onset and episodic course}

The semi-structured interview included the following item 'How much time has passed between the onset of the first symptom to the time at which your symptoms peaked (during that episode)' with the following four possible response alternatives: $<24 \mathrm{~h}, 24-$ $72 \mathrm{~h}, 4-14$ days or $>2$ weeks. Participants were also asked to specify onset time in an open-ended question. All participants rated the general course of their disorder by selecting one of nine alternatives: (a) one flare with remission, (b) chronic course, (c) progressive course, (d) two or more episodes with remission in between, (e) progressive course with flares, (f) initial flare with remission and subsequent progression, (g) severe symptoms at onset and gradual improvement, (h) two or more flares with improvement of baseline function or (i) other course, please describe. The alternatives were also shown as graphs of progress (shown in supplementary Fig. 2).

In addition to the PANS Scale-R, we developed our own instrument in order to determine symptom load at onset and investigate the presence of an episodic course. Two questionnaires were developed; The PANS/PANDAS Related Symptom Inventory (PPRSI) and The Signs of Severity Questionnaire (SOSQ). The PPRSI includes 15 PANS- and PANDAS-related symptoms, including those of OCD, tics and eating disorders. The SOSQ is a measure of 18 other severe psychiatric symptoms (i.e. not specifically related to PANS), foremost suicidal, violent, and psychotic symptoms (see supplementary Files 1 and 2 for the complete instruments).

The structure is identical in both the PPRSI and SOSQ (i.e. assessment of present symptoms, their progression and the course). Each individual symptom is rated as present or never present. If present, the occurrence is recorded to be present 'before onset', 'at onset' or 'after onset' of the main current psychiatric problem (i.e. PANS if relevant). Each symptom could be rated as present at several occasions (for example both 'at onset' and 'after onset'). The course of each symptom was recorded to be 'one flare', 'several flares', 'weekly', or 'fluctuating' and a 'flare' was defined as a sudden and dramatic exacerbation of the symptom. The responses were dichotomised into two categories for the statistical analysis: 'episodic course' (i.e. 'one flare' or 'several flares'), and 'non-episodic course' (i.e. 'every week' or 'fluctuating'). Categories are exclusive (i.e. only one type of course is recorded for each symptom).

\section{Statistical analysis and data management}

All statistical analyses were conducted using SPSS (version 23) and Microsoft Excel. In order to test if the three groups had similar demographic characteristics, continuous and ordinal variables were compared using one-way ANOVAs. Categorical variables were compared using $\chi^{2}$-tests. We used the Bonferroni method for controlling for multiple testing within each analysis.

Symptom load at disorder onset was measured using the PPRSI and SOSQ. We calculated the total number of symptoms for each group at each time, then we compared the relative proportions of symptoms 'before first onset', 'at first onset', and 'after onset' between the three groups using a $3 \times 3 \chi^{2}$-test.

Episodic course was also measured using the PPRSI and SOSQ. We calculated the total number of symptoms reported to be 'episodic' and 'non-episodic' for each group. The difference in proportion between 'episodic' and 'non-episodic' symptoms was analysed using a $2 \times 3 \chi^{2}$-test.

\section{Ethical considerations}

The authors assert that all procedures contributing to this work comply with the ethical standards of the relevant national and institutional committees on human experimentation and with the Helsinki Declaration of 1975, as revised in 2008. All procedures involving patients were approved by the Regional Ethics Review Board of Stockholm (2014/551-31/2; 2014/1711-32; 2015/964-31, 2016/2121-32). All study participants and/or legal guardians granted informed consent. The family who provided the anonymised timeline shown in supplementary Fig. 7 provided additional informed consent for publication.

\section{Results}

\section{Participant classification and demographics}

A total of 28 participants fulfilled criteria for PANS or PANDAS and, as such, comprise the IC-PANS group (24 were from the Cunningham Panel sample and 4 from the Örebro sample). The remaining 29 participants from the Cunningham Panel sample did not meet PANS criteria and subsequently comprise the S-PANS group. Thirty-two of the participants in the Örebro sample did not meet the PANS criteria and thus constitute the never-PANS group. One participant, from the Örebro sample, reported an onset of more than $72 \mathrm{~h}$ between first and full 
symptoms during the interview. Nevertheless, this patient was classified as having PANDAS because of several other clinical signs, including a temporal relationship between streptococcal infection and symptom exacerbations, and was therefore classified as having IC-PANS.

The three groups differed in age and gender; the never-PANS group was older $(P=0.04)$ and the S-PANS group included fewer females $(28 \%, n=8)$ than the other two groups $(50 \%(n=14)$ and $59 \%(n=19)$, respectively, $P=0.007)$. At the time of assessment, the S-PANS group reported more disabling symptoms, according to CGI-S (mean 5.33, s.d. =1.4), compared with the IC-PANS group (mean 4.41, s.d. $=1.7$ ) and the never-PANS group (mean 4.17 , s.d. $=1.7, P=0.02$ ). There were no differences between groups regarding the measures of inflammation and no group had elevated C-reactive protein or erythrocyte sedimentation rate. Similarly, BMI was similar between groups and ranged from 14.8 to 48.4 (See Table 1 for full details of demographic measures).

According to the MINI, all three groups had a similar number of lifetime diagnoses. The two most common current diagnoses were, in the IC-PANS group OCD $(71 \%, n=20)$ and Tourette syndrome $(37 \%, n=10)$; in the S-PANS group OCD $(61 \%, n=17)$ and pervasive developmental disorder $(59 \%, n=17)$; and in the never-PANS group generalised anxiety disorder $(44 \%, n=14)$ and pervasive developmental disorder $(40 \%, n=12)$ (see Table 2 for details).

\section{PANS symptoms, PANS Scale-R}

There were no differences between patients in the S-PANS and ICPANS groups in frequency of the symptoms measured with the PANS Scale-R. However, the never-PANS group reported fewer symptoms than the other two groups. In total, $89 \%(n=25)$ of the participants in the IC-PANS group reported obsessive-compulsive symptoms, compared with $83 \%(n=24)$ in the S-PANS group and $44 \%(n=14)$ in the never-PANS group. The most common obsessive-compulsive symptom reported in the IC-PANS group was contamination fears, whereas symmetry symptoms were more common in the S-PANS and never-PANS groups.

When using the Bonferroni method for correcting for multiple testing, six symptoms were more common in the IC-PANS and S-PANS groups than in the never-PANS group: any obsessive-compulsive symptom, separation anxiety, behavioural regression, loss of academic skills, dilated pupils and simple motor tics. The three groups reported similar frequency of general anxiety (total $57 \%$, $n=49$ ), panic episodes (total $42 \%, n=36$ ), depression (total $68 \%$, $n=59$ ), attention-deficit (total $78 \%, n=67$ ) and hallucinations (total 36\%, $n=29$ ) (Table 3).

\section{Symptom load at disorder onset}

Acute onset was more common in the IC-PANS group (96\%, $n=$ 27) than in the S-PANS group $(17 \%, n=5)$ and in the neverPANS group $(3 \%, n=1)\left(\chi^{2}=99.75\right.$, d.f. $\left.=2, P<0.001\right)$. This was expected and is a result of the diagnostic criteria. As measured with the PPRSI, both the S-PANS and the IC-PANS groups reported most PANS-related symptoms at onset (55\% $(n=204 / 369$ symptoms) and $49 \%$ ( $n=174 / 352$ symptoms), respectively), whereas the never-PANS group reported most PANS-related symptoms after onset $(49 \%, n=102 / 285$ symptoms $)\left(\chi^{2}=32.28\right.$, d.f. $=4, P<$ 0.001) (see Fig. 1 for details).

Contrarily, the onset of severe psychiatric symptoms, as measured with the SOSQ, provided a different pattern. The IC-PANS group reported more symptoms at onset than the other two groups $\left(\chi^{2}=11.00\right.$, d.f. $\left.=4, P=0.02\right)$, but most of the severe symptoms developed after onset in all three groups (see Fig. 1 for details). Interestingly, 36\% $(n=10)$ of the IC-PANS group reported suicidal ideation at onset, which was more frequent than the S-PANS $(10 \%, n=3)$ and never-PANS $(6 \%, n=2)$ groups $\left(\chi^{2}=10.55\right.$, d.f. $=4$, $P<0.01)$. All three groups reported similar rates of suicidal ideation 'after onset' $(29 \%(n=8), 31 \%(n=10)$ and $34 \%(n=10)$, respectively). Detailed data is available in supplementary Figs. 3 and 4.

\section{Episodic course}

We assessed the presence of an episodic course using a question in which participants were asked to choose one out of nine possible courses (shown in supplementary Fig. 2). A total of 32 participants (IC-PANS $n=12$, S-PANS $n=8$, never-PANS $n=12$ ) chose to describe their symptom course as 'other'. Therefore, this question cannot be used to analyse different disorder patterns between groups. Nevertheless, many of the participants who described their disorder as 'other' chose to draw a graph of their own, in order to illustrate their view of the timeline of the disorder. An example of such a timeline is shown in supplementary Fig. 7. Many participants also reported that different types of symptoms ran different courses, with some symptoms being chronic and others appearing in flares.

When assessing the course of each symptom, we acquired a more complete data-set than when asking for a global assessment of course using the timeline. The IC-PANS group reported 39\% ( $n=111 / 283$ symptoms) of their lifetime PANS-related symptoms tended to follow an episodic course, in contrast to the S-PANS $(22 \%, n=54 / 249)$ and the never-PANS groups $(13 \%, n=26 / 195)$ $\left(\chi^{2}=19.03\right.$, d.f. $\left.=2, P<0.001\right)$. A similar pattern was found for the symptom course of severe psychiatric symptoms measured with the SOSQ, in which the IC-PANS group reported an episodic

\begin{tabular}{|c|c|c|c|c|c|c|c|}
\hline & $\begin{array}{l}\text { Interview-confirmed } \\
\text { PANS }(n=28)\end{array}$ & $\begin{array}{c}\text { Suspected } \\
\text { PANS }(n=29)\end{array}$ & $\begin{array}{l}\text { Not suspected } \\
\text { PANS }(n=32)\end{array}$ & $\begin{array}{l}\text { Missing, } \\
n\end{array}$ & $F$ & d.f. & $P$ \\
\hline Age, mean (s.d.) & $16.07(7.3)$ & $14.41(7.7)$ & $21.02(9.5)$ & - & 3.36 & 2,86 & 0.04 \\
\hline Female, $n(\%)$ & $14(50)$ & $8(28)$ & $19(59)$ & - & 5.20 & 2,86 & 0.007 \\
\hline Acute onset $<72 \mathrm{~h}, n(\%)$ & $27(96)$ & $5(17)$ & $1(3)$ & 2 & 99.76 & 2,84 & $<0.001$ \\
\hline Y-BOCS or CY-BOCS, mean (s.d.) & $16.8(12.4)$ & $13.7(13.4)$ & $5.2(8.4)$ & 16 & 7.09 & 2,70 & 0.02 \\
\hline CGI-S at time of assessment (mean, s.d.) & $4.41(1.7)$ & $5.33(1.4)$ & $4.17(1.7)$ & 5 & 4.10 & 2,81 & 0.02 \\
\hline $\begin{array}{l}\text { Number of diagnoses according to MINI, } \\
\text { mean (s.d.) }\end{array}$ & $5.1(4.7)$ & $6.5(4.2)$ & $4.5(2.8)$ & - & 2.54 & 2,86 & 0.08 \\
\hline Body mass index & $23.5(7.7)$ & $21.5(5.6)$ & $24.8(7.4)$ & 34 & 0.91 & 2,52 & 0.41 \\
\hline Plasma C-reactive protein, mean, s.d. & $1.08(2.0)$ & $1.3(2.5)$ & $2.2(3.9)$ & 16 & 0.91 & 2,70 & 0.40 \\
\hline $\begin{array}{l}\text { Blood erythrocyte sedimentation rate, } \\
\text { mean (s.d.) }\end{array}$ & $7.31(5.3)$ & $5.68(4.0)$ & $5.64(8.6)$ & 26 & 0.36 & 2,60 & 0.7 \\
\hline Estimated IQ (mean, s.d.) & $98(21)$ & $85(20)$ & $91(21)$ & 27 & 2.02 & 2,59 & 0.14 \\
\hline
\end{tabular}


Table 2 Diagnoses according to Mini International Neuropsychiatric Interview (MINI) and MINI-KID

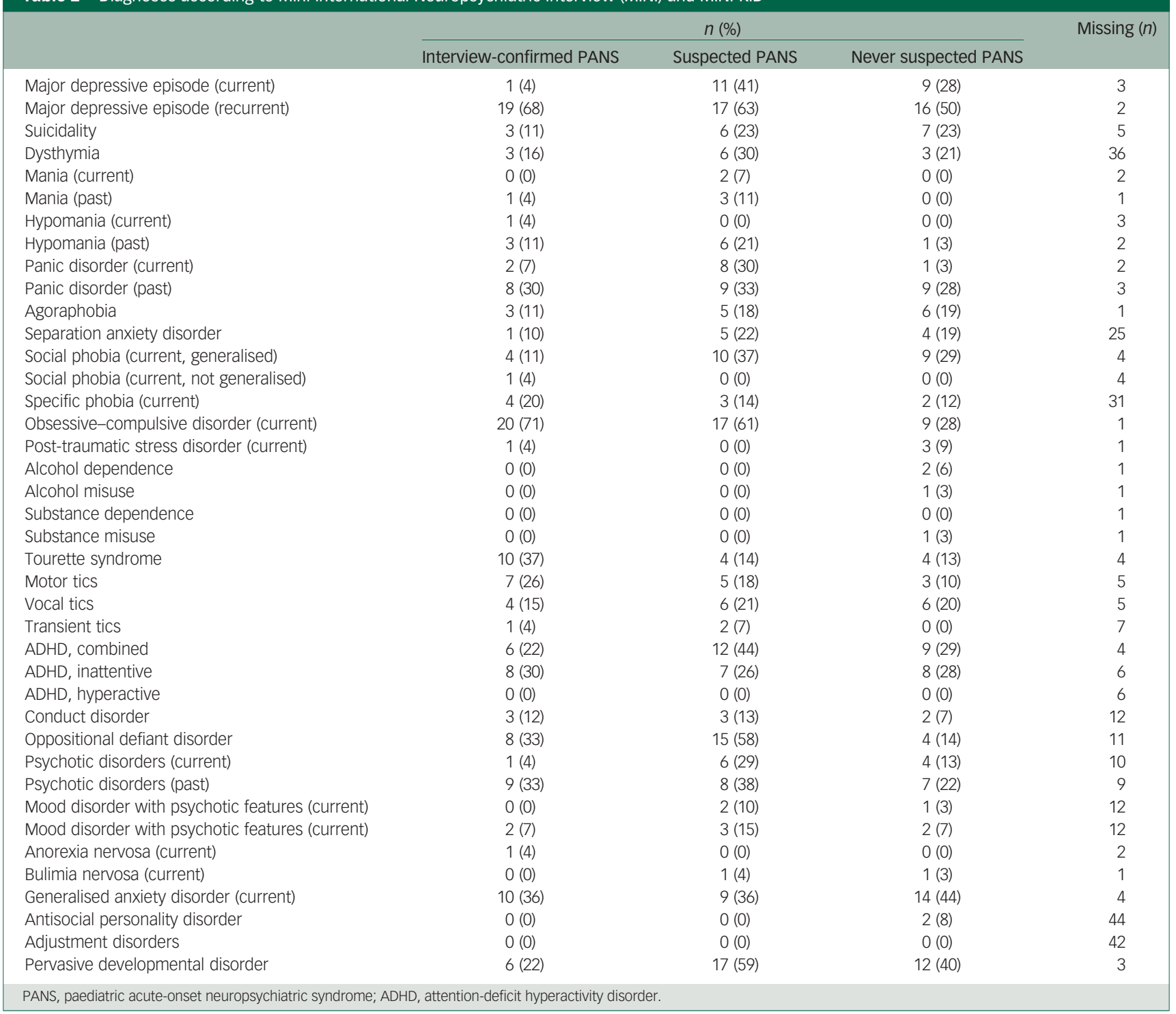

course in $58 \%(n=71 / 122)$ of lifetime symptoms, compared with $27 \%(n=34 / 125)$ in the S-PANS group and $28 \%(n=31 / 109)$ in the never-PANS group $\left(\chi^{2}=31.47\right.$, d.f. $\left.=2, P<0.001\right)$ (see Fig. 1 for details).

Lifetime suicidal ideation was reported by 17 participants (61\%) in the IC-PANS group compared to 13 participants (45\%) in the S-PANS group and 13/32 (41\%) in the never-PANS group, despite the IC-PANS group being younger $\left(\chi^{2}=2.62\right.$, d.f. $\left.=2, P=0.27\right)$. Suicidal ideation was more commonly reported to follow an episodic course in the IC-PANS group (65\%, $n=11 / 17$ participants) than in the S-PANS $(38 \%, n=5 / 13$ participants) or the never-PANS $\left(15 \%, n=2 / 13\right.$ participants) groups $\left(\chi^{2}=8.09\right.$, d.f. $\left.=2, P=0.02\right)$. More detailed information is presented in supplementary Figs 5 and 6.

\section{Discussion}

The aim of this study was to describe a group of Swedish patients with PANS and to compare the onset and course of their symptoms with those of patients with psychiatric conditions other than PANS. A total of 89 patients with psychiatric illness were included in the study. They were classified into three groups: (a) confirmed to have PANS; (b) previously suspected to have PANS but failed to meet the full criteria; or (c) never suspected to have PANS. Patients with IC-PANS reported a higher proportion of symptoms that follow an episodic course than the other two groups. Additionally, participants with both IC-PANS and S-PANS reported more symptoms at disorder onset. The diagnostic criterion most important for classifying PANS was 'acute onset'. Thus, we conclude that the acute onset of a psychiatric disorder is associated with a high symptom load at disorder onset and with an episodic course. These three disorder characteristics are suggestive of PANS.

\section{The acute-onset criterion of PANS and PANDAS}

In the current study, an acute onset (less than $72 \mathrm{~h}$ ) of OCD, eating restrictions and/or tics, was the most important criterion that defined the IC-PANS group. However, we found it difficult to assess the onset because of differences in the onset pattern for the different symptoms that characterise PANS. Some participants reported a vague prodromal phase (which was also noted in the report by Swedo et al of the first 50 cases $^{1}$ ), whereas others reported an acute onset, but only for a subset of symptoms. However, during the interview, it was up to the clinician to decide, based on all available information, whether or not the onset was acute or not. 
Table 3 PANS Scale-R - lifetime paediatric acute-onset neuropsychiatric syndrome (PANS) symptoms ${ }^{a}$

\begin{tabular}{|c|c|c|c|c|c|c|c|c|}
\hline & \multirow[t]{2}{*}{$\begin{array}{l}\text { Interview-confirmed } \\
\text { PANS }(n=28)\end{array}$} & \multirow[t]{2}{*}{$\begin{array}{l}\text { Suspected PANS } \\
\qquad(n=29)\end{array}$} & \multirow[t]{2}{*}{$\begin{array}{l}\text { Never suspected } \\
\text { PANS }(n=32)\end{array}$} & \multirow[t]{2}{*}{$\begin{array}{l}\text { Missing } \\
\quad(n)\end{array}$} & \multicolumn{4}{|c|}{$\begin{array}{c}\text { Comparison between group } \\
\text { frequencies }\end{array}$} \\
\hline & & & & & $\chi^{2}$ & $F$ & d.f. & $P$ \\
\hline \multicolumn{9}{|l|}{ Obsessive-compulsive symptoms, ${ }^{\mathrm{b}} n$ (\%) } \\
\hline Any obsessive-compulsive symptom & 25 (89) & $24(83)$ & $14(44)$ & 1 & 16.736 & & 2 & $<0.001$ \\
\hline Contamination & $17(61)$ & $16(55)$ & 7 (23) & 1 & 10.276 & & 2 & 0.006 \\
\hline Causing harm & $13(46)$ & $9(31)$ & $4(13)$ & 2 & 7.598 & & 2 & 0.022 \\
\hline Sexual or religious & $7(25)$ & $3(10)$ & $4(13)$ & 2 & 2.524 & & 2 & 0.283 \\
\hline Symmetry & $16(57)$ & $20(69)$ & $9(29)$ & 1 & 10.155 & & 2 & 0.006 \\
\hline Hoarding & $7(25)$ & $10(34)$ & $4(13)$ & 1 & 3.869 & & 2 & 0.144 \\
\hline Other & $7(26)$ & $5(18)$ & $2(7)$ & 6 & 3.488 & & 2 & 0.175 \\
\hline Eating disorder, $n(\%)$ & $17(61)$ & $17(59)$ & $8(26)$ & 1 & 9.243 & & 2 & 0.010 \\
\hline Separation anxiety, $n$ (\%) & $22(79)$ & $19(66)$ & $8(26)$ & 1 & 18.294 & & 2 & $<0.001$ \\
\hline General anxiety, $n$ (\%) & $16(62)$ & $14(48)$ & $19(61)$ & 3 & 1.352 & & 2 & 0.509 \\
\hline Phobias, $n$ (\%) & $13(46)$ & $13(45)$ & $3(10)$ & 1 & 11.753 & & 2 & 0.003 \\
\hline Panic episodes, $n$ (\%) & $12(43)$ & $14(50)$ & $10(34)$ & 4 & 1.409 & & 2 & 0.494 \\
\hline Emotional lability, $n$ (\%) & $20(71)$ & $25(86)$ & $15(52)$ & 3 & 8.228 & & 2 & 0.016 \\
\hline Depression, $n(\%)$ & $20(71)$ & $19(66)$ & $20(67)$ & 2 & 0.256 & & 2 & 0.880 \\
\hline Irritability or aggression, $n$ (\%) & $18(64)$ & $24(83)$ & $15(50)$ & 2 & 7.032 & & 2 & 0.030 \\
\hline Behavioural regression, $n$ (\%) & $13(46)$ & $19(66)$ & $3(10)$ & 2 & 19.561 & & 2 & $<0.001$ \\
\hline Personality change, $n(\%)$ & $17(61)$ & $19(66)$ & $7(23)$ & 2 & 12.602 & & 2 & 0.002 \\
\hline Attention deficit, $n(\%)$ & $20(71)$ & $25(89)$ & $22(73)$ & 3 & 3.154 & & 2 & 0.207 \\
\hline Loss of academic skills, $n$ (\%) & $17(63)$ & $13(52)$ & $3(10)$ & 8 & 17.933 & & 2 & $<0.001$ \\
\hline Sensory sensitivity, $n(\%)$ & $23(82)$ & $25(86)$ & $16(53)$ & 2 & 9.757 & & 2 & 0.008 \\
\hline Hallucinations, $n(\%)$ & $9(36)$ & 9 (33) & $11(39)$ & 9 & 0.212 & & 2 & 0.900 \\
\hline Dilated pupils, $n$ (\%) & $13(46)$ & $14(48)$ & $1(4)$ & 5 & 15.741 & & 2 & $<0.001$ \\
\hline Urinary symptoms, $n$ (\%) & $21(75)$ & $17(59)$ & $9(31)$ & 3 & 11.389 & & 2 & 0.003 \\
\hline Dysgraphia, $n(\%)$ & $12(44)$ & $15(56)$ & $6(20)$ & 5 & 7.976 & & 2 & 0.019 \\
\hline Choreiform movements, $n$ (\%) & $11(42)$ & $13(46)$ & $3(11)$ & 8 & 9.103 & & 2 & 0.011 \\
\hline Complex motor tics, $n(\%)$ & $10(37)$ & $11(38)$ & $3(10)$ & 3 & 7.348 & & 2 & 0.025 \\
\hline Motor hyperactivity, n (\%) & $13(46)$ & $20(69)$ & $9(30)$ & 2 & 9.023 & & 2 & 0.011 \\
\hline Simple motor tics, $n$ (\%) & $20(71)$ & $13(45)$ & $7(23)$ & 2 & 13.511 & & 2 & 0.001 \\
\hline Sleep problems, $n(\%)$ & 25 (89) & $23(79)$ & $19(63)$ & 2 & 5.64 & & 2 & 0.060 \\
\hline Global severity 0-100, $n$ (mean) s.d. & $27(48.74) 26.2$ & $27(65.74) 22.0$ & $27(42.17) 29.7$ & 8 & & $F=5.83$ & 2,78 & 0.004 \\
\hline
\end{tabular}

We chose to classify one patient from the Örebro sample as having PANS despite a report by the parents of a subacute (4-14 days) onset. The family reported during the interview for this study that the onset was gradual, but in the medical records the family had reported an acute onset with concomitant hoarding behaviours, separation anxiety and hallucinations. Streptococcal infections preceded both the onset and subsequent exacerbations of symptoms. When taking all available information into account, the final decision was that this participant fulfilled criteria for PANDAS and was thereby included in the IC-PANS group. The conflicting information of the interview and the medical records illustrate the difficulty that clinicians face when assessing the acute onset of PANS.

Different studies have used slightly different methods to define PANS, which may hinder comparisons. Analogous to our definition for categorising PANS, the sudden-onset criterion was used in a study at a multidisciplinary PANS clinic. ${ }^{5}$ For a diagnosis of PANDAS, a relationship between the onset, exacerbation of symptoms and a streptococcus infection is mandatory, and thus a temporal association with streptococcal infection was the main definition for inclusion in an early study of PANDAS that included individuals where the onset was insidious. ${ }^{23}$

\section{Symptom load at onset and episodic course, PPRSI and SOSQ}

One way of measuring the severity of a disorder is to quantify the load of symptoms that are related to the disorder itself. We enabled measurement of the load of PANS-related symptoms by developing a questionnaire for this particular purpose, the PPRSI.
We also wanted to measure other severe psychiatric symptoms that are not specifically related to PANS, but are sometimes described in the literature, and did so by constructing the SOSQ scale. Both patients in the IC-PANS group and those in the SPANS group received higher scores than those in the never-PANS group at disorder onset, which illustrates the dramatic and overwhelming start of this disorder.

The assessment of episodic course proved difficult. When we asked the participants to describe the course and exacerbations of the symptoms, we gave them examples on different types of timelines in a graph. However, most patients felt that the graphs did not fit their own experiences and instead chose the option 'other' and drew a timeline of their own. The timeline illustrates the onset and exacerbations of symptoms and creates a mutual base for understanding what and when things have happened to the patient. In supplementary Fig. 7, we provide an example of a patient timeline, which spans over several years and includes information about severity, symptoms and treatment. Our experience from this study is that the timeline was often helpful, assisting the participant in remembering details of their disorder and its course.

We were more successful in obtaining quantitative data on episodic course by asking the participants to rate whether each symptom was episodic or non-episodic. By using this method, we can show that patients with confirmed PANS, more often than other patients, report an episodic course. Thus, patients with PANS not only differ from the other groups by the sudden onset of symptoms, but also by experiencing a larger proportion of symptoms present only in flares. Notably, our patients with IC-PANS tended to experience suicidal ideation at disorder onset and during flares, in contrast to the reports of the other two groups. 
(a)

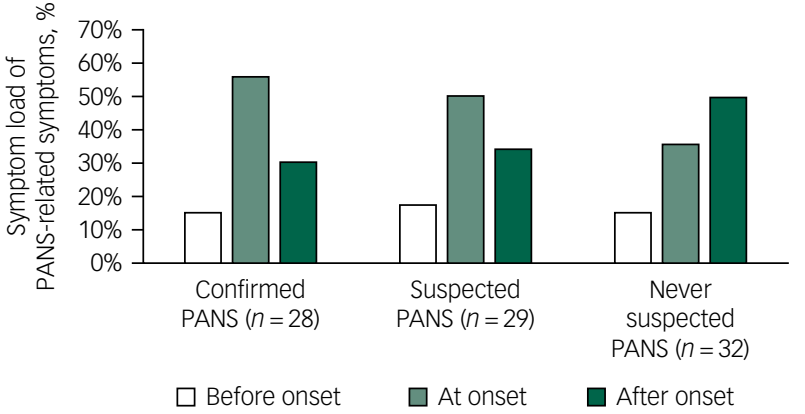

(c)

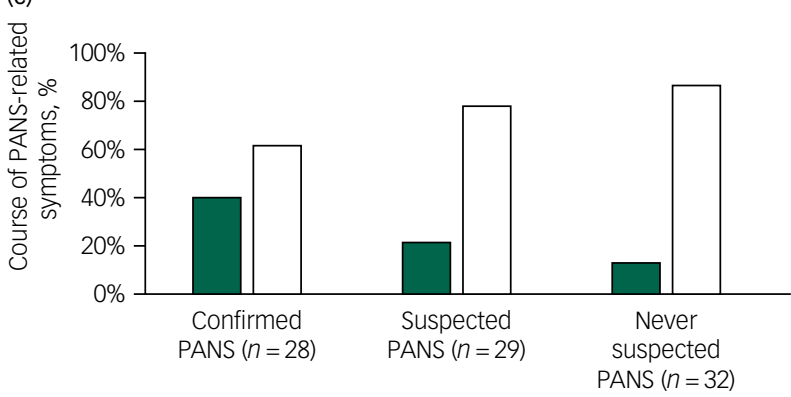

Episodic course (b)

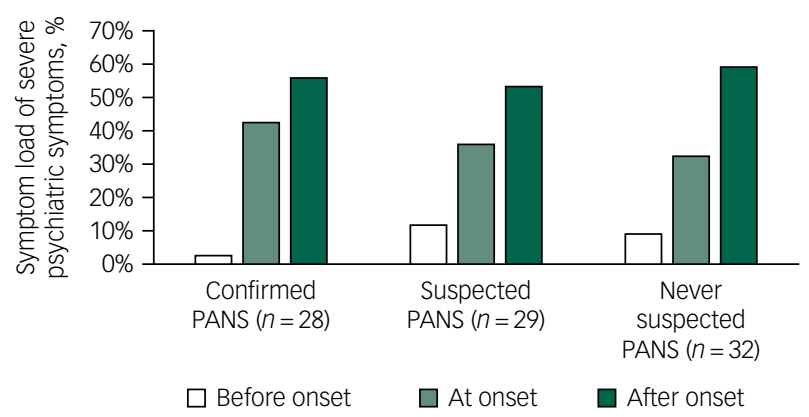

(d)

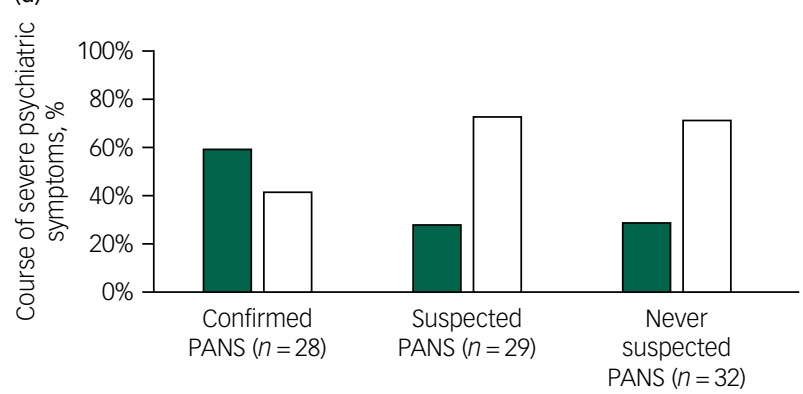

Episodic course

\section{Fig. 1 Symptom load at disorder onset and episodic course.}

(a) Time of onset of paediatric acute-onset neuropsychiatric syndrome (PANS)-related symptoms measuring with the PANS/PANDAS Related Symptom Inventory (PPRSI) and (b) time of onset of severe psychiatric symptoms measured using the Signs of Severity Questionnaire (SOSQ). Each symptom could be rated as present at several times (i.e. both before and after onset). Disorder onset was defined during the interview. (c) and (d) Show the proportion of present symptoms reported to have an episodic versus non-episodic course. (c) Shows PANS-related symptoms measured with the PPRSI and (d) shows severe psychiatric symptoms measured with the SOSQ. Each symptom was reported either as 'episodic' or 'non-episodic'. PANDAS, paediatric autoimmune neuropsychiatric disorders associated with streptococcal infections.

\section{PANS symptoms, PANS Scale-R}

The IC-PANS and S-PANS groups did not differ from each other on any measure of the PANS Scale-R, except for the duration of onset, consistent with previous reports. ${ }^{5}$ When we compare our results with a study by Murphy et al, ${ }^{24}$ our patients had similarly high scores on ratings of separation anxiety, OCD and tics; however, our patients presented less behavioural regression ( $46 \%$ compared with $84 \%$ ) and deterioration of school performance (63\% compared with $88 \%$ ). Dilated pupils were, however, more often reported by our participants with PANS compared with Murphy et al's participants. Comorbidity was present for all participants, with sleep and anxiety disorders being particularly common. Interestingly, common psychiatric symptoms not specifically related to PANS (for example depression, anxiety and hallucinations) were equally present in all three groups. The symptoms in the PANS Scale-R, and thereby the symptoms of criterion II for PANS, did not differentiate between confirmed and suspected cases. Moreover, many of the same symptoms were also common in the psychiatric patient comparison group.

\section{Limitations}

The main limitation of this study is the lack of validated instruments to assess the main outcome measures (i.e. PANS related symptoms, onset duration and episodic course). Many psychiatric assessment tools are aimed at establishing diagnosis without using differential diagnostics. Many tools also assess symptoms within a specific time frame (for example the past 2 weeks), which is clinically useful, but unhelpful in the work-up for PANS. The PANS specific tools developed by us for the purpose of this study and the PANS Scale-R developed by Leckman are difficult and time consuming to administer. Nevertheless, they are valuable tools since there are no existing validated scales to assess onset, episodic course or symptoms present at onset. By publishing our working materials in the supplement, we hope to be part of a much-needed effort to standardise PANS assessment. We have recently developed an instrument to assess PANS (the PsychoNeuroInflammatory related Signs and Severity Inventory ${ }^{25}$ ), which is currently being validated in an independent sample.

Additionally, we did not examine the patients with PANS at the time of onset and therefore our data is based on retrospective interviews and aided by medical records when available. As treatment is usually given when patients seek medical attention, the retrospective definition of what is 'a flare' and what is a recurrent psychiatric condition can be difficult to disentangle. The risk of recall bias because of information in the press or social media may also be high for nonestablished diagnoses such as PANS and PANDAS. However, the 53 patients previously assessed for PANDAS (in the Cunningham Panel sample) were recruited prior to June 2014, prior to PANS and PANDAS receiving media attention in Sweden. A strength of our retrospective approach is that it enabled us to assess the long-term course, which would not have been possible if we only examined the participants at onset.

Unfortunately, we failed to recruit enough young children with psychiatric disorders in the Örebro comparison sample. Therefore, the patients in the Örebro sample (and thereby the never-PANS group) are older than the other two groups. The difference in age of the groups possibly influenced our results by introducing recall bias of a different nature in the groups, and because all measures of disorders present at any time in the life of the patients are expected to increase with age. Another strength of our study is, nevertheless, this comparison sample of patients with psychiatric conditions with a similar level of overall disability as the Cunningham Panel sample (CGI-S mean of 4.9 v. 4.2, not significant). 
Our findings show that the Cunningham Panel sample had more lifetime psychiatric symptoms than the Örebro comparison sample. This raises the question if there was bias regarding which of the eligible patients $(n=154)$ chose to participate in the study. As the laboratory made the invitation to participate in the study, the identity of the non-participants is unknown to us, and we cannot know if the individuals who chose to participate were different in age, gender or severity than those who chose not to participant. We do know, however, that the participants and nonparticipants had similar responses on the Cunningham Panel. ${ }^{16}$ Also, as the Örebro sample included patients with many different psychiatric disorders, not just OCD, tics or eating disorders, we cannot tell if patients with S-PANS have more symptoms than patients with non-PANS OCD, tics or eating disorders, which is also a limitation of our study design.

Similar to other studies on cohorts with PANS and PANDAS, our study suffers from a circular argument; specifically, we include patients suspected of having PANS and PANDAS and then conclude that they are similar. This is a consequence of selection, in that they were selected because they were similar to other patients with PANS or PANDAS. This methodical problem constitutes a recognised dilemma when describing any new clinical entity. However, the inclusion criterion was to previously have taken the Cunningham Panel (ordered by the treating physician and not by us), which possibly ameliorated this bias.

Some of the differences between the groups are artefacts related to our inclusion process. The Cunningham Panel sample had previously been ill enough to warrant an examination using the Cunningham Panel, but at the time of assessment, some participants were considerably improved. Contrary to this, the main inclusion criterion for the Örebro sample was to have a current psychiatric disorder at the time of our assessment. Thus, the Örebro sample, by definition, must have symptoms 'after onset' (i.e. have symptoms at the time of our assessment), whereas individuals in the Cunningham Panel sample could report most symptoms 'at onset'. However, our finding that an episodic course was more common in the IC-PANS group compared with the other patients does not confirm the above-mentioned bias as specifying course was not a requirement for inclusion in any of the groups.

\section{Implications}

In this study, confirmed PANS (i.e. acute onset) was associated with a higher load of symptoms at onset, often including suicidal ideations and the presence of an episodic course. The symptom panorama was, aside from the pattern of onset and the presence of episodic course, almost indistinguishable in the IC-PANS and S-PANS groups. Although the PANS criteria do not require exacerbation of symptoms or flares, our results nevertheless confirm that acute-onset PANS is often accompanied by a high symptom load at onset and by an episodic course. When assessing and diagnosing PANS, the focus should be on the onset and course of the disorder, rather than on individual psychiatric symptoms.

Eva Hesselmark, BSc (D), PhD Student, Department of Clinical Neuroscience, Center for Psychiatry Research, Karolinska Institutet; and Stockholm Health Care Services, Stockholm County Council, Sweden; Susanne Bejerot, MD, PhD (iD), Professor, schoo of Medical Sciences, Örebro University; University Health Care Research Center, Faculty of Medicine and Health, Örebro University; and Department of Clinical Neuroscience, Center for Psychiatry Research, Karolinska Institutet, Sweden

Correspondence: Eva Hesselmark, CAP Research Center, Karolinska Institutet, Gävlegatan 22, 8tr, 11330 Stockholm, Sweden. Email: eva.hesselmark@ki.se

First received 19 Sep 2018, final revision 30 Jan 2019, accepted 31 Jan 2019

\section{Funding}

This research was funded by grants from the Swedish Research Council (S. B. Grant number: 523-2011-3646), Hjärnfonden (S.B. Grant number: FO2015-0191), Bror Gadelius Minnesfond (E.H.), Psykiatrifonden (E.H.), and by grants provided by the Stockholm County Council (S.B. Grant numbers: PPG projects 20130671 and 20150150). The funding sources had no influence over the study design, collection or interpretation of data or any other part of the research process.

\section{Acknowledgements}

We wish to warmly thank the participants in this study and their families. We also thank Sara Ekman, Ulrika Hylén, Karin Lobenius Palmér, Jasmina Popaja and Machi Cleantous for assisting with data management and data collection, as well as Wieslab for assistance with patient recruitment. This study is based on data collected within the project 'PANS - A detailed study of the patients, their symptoms, biomarkers and treatment offered in a Scandinavian cohort', which was registered prior to enrolment of any participants; http://clinicaltrials.gov; NCT02190292.

\section{Supplementary material}

Supplementary material is available online at https://doi.org/10.1192/bjo.2019.10.

\section{References}

1 Swedo SE, Leonard HL, Garvey M, Mittleman B, Allen AJ, Perlmutter S, et al. Pediatric autoimmune neuropsychiatric disorders associated with streptococcal infections: clinical description of the first 50 cases. Am J Psychiatry 1998; 155: 264-71.

2 Swedo SE, Leckman JF, Rose NR. From research subgroup to clinical syndrome: modifying the PANDAS criteria to describe PANS (Pediatric Acute-onset Neuropsychiatric Syndrome). Pediatr Ther 2012; 2: 113.

3 Frankovich J, Swedo S, Murphy T, Dale RC, Agalliu, Danes M, et al. Clinical management of pediatric acute-onset neuropsychiatric syndrome: Part II—use of immunomodulatory therapies. J Child Adolesc Psychopharmacol 2017; 27: 574-93.

4 Sigra S, Hesselmark E, Bejerot S. Treatment of PANDAS and PANS: a systematic review. Neurosci Biobehav Rev 2018; 86: 51-65.

5 Frankovich J, Thienemann M, Pearlstein J, Crable A, Brown K, Chang K. Multidisciplinary clinic dedicated to treating youth with pediatric acute-onset neuropsychiatric syndrome: presenting characteristics of the first 47 consecutive patients. J Child Adolesc Psychopharmacol 2015; 25: 38-47.

6 Brown K, Farmer C, Farhadian B, Hernandez J, Thienemann M, Frankovich J. Pediatric acute-onset neuropsychiatric syndrome response to oral corticosteroid bursts: an observational study of patients in an academic community-based PANS clinic. J Child Adolesc Psychopharmacol 2017; 27: 629-39.

7 Brown KD, Farmer C, Freeman GM, Spartz EJ, Farhadian B, Thienemann M, et al. Effect of early and prophylactic nonsteroidal anti-inflammatory drugs on flare duration in pediatric acute-onset neuropsychiatric syndrome: an observational study of patients followed by an academic community-based pediatric acuteonset neuropsychiatric syndrome clinic. J Child Adolesc Psychopharmacol 2017; 27: 619-28.

8 Chang K, Frankovich J, Cooperstock M, Cunningham MW, Latimer ME, Murphy TK, et al. Clinical evaluation of youth with pediatric acute-onset neuropsychiatric syndrome (PANS): Recommendations from the 2013 PANS consensus conference. J Child Adolesc Psychopharmacol 2015; 25: 3-13.

9 Gilbert DL, Mink JW, Singer HS. A Pediatric neurology perspective on pediatric autoimmune neuropsychiatric disorder associated with streptococcal infection and pediatric acute-onset neuropsychiatric syndrome. J Pediatr 2018; 199: 243-51

10 Moleculera Labs. PANS and PANDAS Diagnosis and Treatment. Moleculera Labs, 2016 (http://www.moleculeralabs.com/pandas-pans-diagnosis-andtreatment/).

11 Cox CJ, Zuccolo AJ, Edwards EV, Mascaro-Blanco A, Alvarez K, Stoner J, et al. Antineuronal antibodies in a heterogeneous group of youth and young adults with tics and obsessive-compulsive disorder. J Child Adolesc Psychopharmacol 2015; 25: 76-85.

12 Kirvan CA, Swedo SE, Heuser JS, Cunningham MW. Mimicry and autoantibodymediated neuronal cell signaling in Sydenham chorea. Nature Med 2003; 9: 914-20.

13 Kirvan CA, Cox CJ, Swedo SE, Cunningham MW. Tubulin is a neuronal target of autoantibodies in Sydenham's chorea. J Immunol 2007; 178: 7412-21.

14 Kirvan CA, Swedo SE, Snider LA, Cunningham MW. Antibody-mediated neuronal cell signaling in behavior and movement disorders. I Neuroimmunol 2006; 179: 173-9. 
15 Singer HS, Mascaro-Blanco A, Alvarez K, et al. Neuronal antibody biomarkers for Sydenham's chorea identify a new group of children with chronic recurrent episodic acute exacerbations of tic and obsessive compulsive symptoms following a streptococcal infection. PLoS One 2015; 10: e0120499.

16 Hesselmark E, Bejerot S. Biomarkers for diagnosis of pediatric acute neuropsychiatric syndrome (PANS) - sensitivity and specificity of the Cunningham Panel. J Neuroimmunol 2017; 312: 31-7.

17 Sheehan DV, Lecrubier $\mathrm{Y}$, Sheehan $\mathrm{KH}$, Amorim $\mathrm{P}$, Janavs J, Weiller $\mathrm{E}$, et al. The Mini-International Neuropsychiatric Interview (M.I.N.I.): the development and validation of a structured diagnostic psychiatric interview for DSM-IV and ICD-10. J Clin Psychiatry 1998; 59 (suppl 20): 22-33.

18 Sheehan DV, Sheehan $\mathrm{KH}$, Shytle RD, Janavs J, Bannon $\mathrm{Y}$, Rogers JE, et al Reliability and validity of the mini international neuropsychiatric interview for children and adolescents (MINI-KID). J Clin Psychiatry 2010; 71: 313-26.

19 Goodman WK, Price LH, Rasmussen SA, Mazure C, Fleischmann RL, Hill CL, et al. The Yale-Brown Obsessive Compulsive Scale. I. Development, use, and reliability. Arch Gen Psychiatry 1989; 46: 1006-11.

20 Guy W. ECDEU Assessment Manual for Psychopharmacology — Revised (DHEW Publ No ADM 76-338). US Department of Health, Education, and Welfare, 1976
21 Wechsler D. Wechsler Adult Intelligence Scale—Fourth Edition Administration and Scoring Manual. Pearson, 2008.

22 Wechsler D. Wechsler Intelligence Scale for Children Third Edition Manual. Psychological Corporation, 1991.

23 Murphy TK, Storch EA, Lewin AB, Edge PJ, Goodman WK. Clinical factors associated with pediatric autoimmune neuropsychiatric disorders associated with streptococcal infections. J Pediatr 2012; 160: 314-9.

24 Murphy TK, Patel PD, McGuire JF, Kennel A, Mutch PJ, Parker-Athill EC, et al. Characterization of the pediatric acute-onset neuropsychiatric syndrome phenotype. J Child Adolesc Psychopharmacol 2014; 25: 14-25.

25 Bejerot S, Hesselmark E, Humble MB. Abstract \# 1786 The PsychoNeurolnflammatory related Signs and Severity Inventory (PNISSI). Brain Behav Immun 2016; 57 (suppl): e23. 